\title{
Development and Pilot Testing of the Transgender and Gender Diverse Fertility Preservation Knowledge Scale
}

\author{
Denise Rivera ${ }^{1}$, Angela Jukkala ${ }^{2}$, Katherine Mistretta ${ }^{3}$, and Willa Starke ${ }^{4}$ \\ 1 Montana State University; denise.rivera2@montana.edu \\ 2 Montana State University; angela.Jukkala@monana.edu \\ 3 Bozeman Creek Family Health; kmistretta@bozemancreekfamilyhealth.com \\ 4 Montana State University; wpstarke@gmail.com \\ * Correspondence: denise.rivera2@montana.edu; Tel.: 1-406-243-6550
}

\begin{abstract}
Little is known about fertility preservation within the TGD community. Few receive adequate counseling placing them at risk for decision regret. The goal of this project was to develop, and pilot test the Transgender Fertility Preservation Knowledge Scale (TFPKS) to support the development and evaluation of health education resources. A community engaged; cross sectional retrospective design was used. Participants $(n=189)$ provided information describing demographics, healthcare decision-making preferences, experiences/knowledge of fertility preservation, and treatment decision regret. The sample included 189 TGD adults. Most were white and aged 26-35 (33.3\%) and not offered a consultation (73.0\%). Many (41.2\%) report they would have participated if offered. Knowledge regarding fertility preservation to support this desire was low. Most participants identified a patient-centered (69.4\%) decision making preference. Much remains to address the healthcare inequities within the TGD population regarding fertility preservation. Overall participants had low levels of knowledge to support decision making. Further, healthcare system and individual barriers to fertility preservation remain prevalent. A foundational step towards addressing these disparities, is the identification of a valid and reliable instrument to measure TGD knowledge of fertility preservation.
\end{abstract}

Keywords: transgender, fertility preservation, decision making preferences

\section{Introduction}

Increasingly individuals are seeking healthcare for gender affirmation services. This treatment frequently includes hormonal therapy and or surgical interventions that may result in permanent sterility. As a result, one of the most critical decisions that must be addressed very early in the treatment program is fertility preservation. Currently, most fertility preservation treatment approaches have been extrapolated from oncology and applied to the transgender and gender diverse (TGD) populations. This approach is problematic in that the lived experience of fertility preservation related to cancer is most likely decidedly different than that related to gender affirmation treatment. The goal of this project was to develop, and pilot test the Transgender Fertility Preservation Knowledge Scale (TFPKS) as a measure of TGD persons knowledge of fertility preservation when pursing gender affirmation treatment.

The true prevalence of TGD identity is unknown ${ }^{1}$; in the United States it is estimated that 1.4 million adults identify as TGD ${ }^{2}$. Simply defined, TGD persons are individuals whose personal gender identity differs from their sex assigned at birth ${ }^{3}$. Greater visibility and acceptance within society is believed to have resulted in greater individual willingness to self-identify at TGD and seek transition healthcare services ${ }^{4}$ creating a demand for high-quality gender affirmation services. Consequently, there has been an 
increase in the number of healthcare professionals and insurers providing coverage for transition related care ${ }^{5}$. While historically, information related to the care and support of the transgender community has received little attention, these two factors have refocused efforts to improve health outcomes within this population.

Though a comprehensive discussion of treatment options specific to fertility preservation is beyond the scope of this manuscript, individual healthcare treatment decisions within the TGD population may vary widely based on personal preferences. As a result, decisions around fertility preservation would be considered preference sensitive, meaning they should be made based upon how treatment options would be experienced by the individual ${ }^{6-8}$. These equipoise healthcare decisions occur in situations where evidence of superiority of one treatment over another is dependent upon how the individual values the risks and benefits of each treatment option ${ }^{9}$. For TGD individuals, treatment options may include no treatment, psychological treatment for gender dysphoria, gender affirming hormone therapy (GAHT) and gender affirmation surgery (GAS) ${ }^{10-12}$. Despite otherwise favorable treatment related health outcomes, the negative impact of affirmation treatment upon future fertility is well documented $7,13-17$. For this reason, high quality health information specific to the risks and benefits of gender affirmation treatment options ${ }^{18}$ should be provided to all patients prior to the initiation of treatment $10,19,20$.

Despite these recommendations, few TGD clients receive adequate counseling or fertility preservation information placing them at risk for decision regret 12,21 . Decision regret has been defined as the experience of distress or remorse following a healthcare decision that is inconsistent with personal values and/or beliefs ${ }^{22}$. While fertility preservation counseling and health information needs within the adolescent and young adult population have been explored ${ }^{21}$, there is limited empirical evidence to guide fertility preservation treatment decision making in TGD adults or health care professionals ${ }^{23,24}$. As such, these resources likely do little to inform individuals following that narrow developmental period. The average age of transition is currently around 30 years of age 6,25 and is a time that may also be characterized by other significant life changes such as developing new relationships and the need to consider partner parenting desires. Health information specific to fertility preservation across childbearing years is needed to ensure informed, high-quality decision making to minimize decision regret.

The concept of parenthood in the TGD client is often variable and individually negotiated with their personal identity 8,26 . Historically, it has been assumed by many that seeking healthcare for gender affirmation eliminated the possibility of biologic children 6,11 and further, that most transgender individuals do not want children ${ }^{11,27}$. However, the reality is that $40 \%$ of transwomen desire to have biologic children and $50 \%$ of transmen want children without regard to biological considerations ${ }^{28-30}$, similar to parenting desires of the general population ${ }^{31}$. The gap between historical disinformation and the lived reality of childbearing desire within the transgender community is troubling and demands attention. Very little is known about fertility preservation and decision regret within the transgender population.

Currently, little is known about how well prepared TGD adults are to make complex, life altering fertility preservation treatment decisions that should be made prior to the initiation of gender affirmation medical treatment. To better understand this phenomenon, we explored transgender adults' experiences with fertility preservation and fertility preservation knowledge prior to initiating gender affirmation treatment. 


\section{Materials and Methods}

The sample included 187 English speaking participants who identified as transmasculine $(n=84 ; 44.7 \%)$, transfeminine $(n=65 ; 34.6 \%)$ or gender diverse $(n=38 ; 20.0 \%)$. Most participants were white $(n=151 ; 81.9 \%)$. Participants completed questions describing demographic background, healthcare decision-making preferences, experiences with and knowledge of fertility preservation, and treatment decision regret.

\section{Measures}

The Transgender Fertility Preservation Knowledge Survey (TFPKS) was cocreated by investigators, endocrinologist, primary care providers, and TGD community partners to gather information describing participant knowledge of fertility preservation as there was no readily available instrument for this purpose. This task was challenging because while some fertility preservation health information knowledge is needed regardless of gender assigned at birth, gender specific information is required as well. In an initial attempt to develop such an instrument, researchers developed the TFPKS to survey participant knowledge of fertility preservation. Content validity was established through literature and expert review by an interprofessional team comprised of researchers, primary care providers, and infertility endocrinologists. Prior to piloting, transfeminine and transmasculine research team members reviewed the TFPKS for acceptability and relevance.

The TFPKS includes 5 items specific to fertility preservation regardless of gender assigned at birth, assessing knowledge specific to topics such as health insurance coverage, healthcare law and the potential impact of gender affirmation treatments upon fertility. Participants who identified as "assigned female at birth" (AFAB) were asked to respond to 5 additional questions specific to female fertility preservation and those assigned "as male at birth" (AMAB) were asked to respond to 2 questions specific to male fertility preservation. A true/false/unsure questionnaire format was used, with unsure responses being coded as "incorrect" for analysis. Participants earned 1 point for each item answered correctly. Points earned by AFAB participants could range from 0-10; points earned by $A M A B$ could range from 0-7.

Decision Making Preferences were measured using the 1 item Control Preferences Scale ${ }^{32}$. This instrument focuses upon the control preferences construct, or the degree of control an individual wants to assume when making healthcare treatment decisions. Having undergone extensive psychometric testing within diverse populations, the Control Preferences Scale has been found to be a valid and reliable measure of an individual's preferences when making healthcare decisions ${ }^{33}$.

Decision Regret Scale was measured using the Decision Regret Scale ${ }^{22}$. This scale was created to measure distress or remorse following the implementation of a healthcare decision and experiencing the resulting health outcomes. With anchors of strongly agree to strongly disagree, this 5-item instrument has been found to be a valid and reliable measure of healthcare decision regret.

\section{Procedure}

A community engaged; retrospective descriptive design was used to gather data from adult transgender individuals who had initiated gender affirmation treatment at least 12 months prior to study participation. The community-engaged (CEnR) approach was utilized to support identification of this community's "truth" 34 through the systematic cocreation of new knowledge ${ }^{35}$. Following university IRB approval, participants were recruited online through social media (Facebook); local, regional, and national LGBTQ support groups; and "snowball sampling" between October and December of 2020. 
Recruitment ads were posted online which contained a hyperlink leading interested individuals to the Qualtrics webpage containing the informed consent and the opportunity to enter a gift card drawing. After providing informed consent and confirming they identified as transgender and had initiated gender affirmation treatment at least 12 months prior to study participation, participants could complete the survey. Study questionnaires was hosted on a secure server administered by the research team's university using the Qualtrics platform.

A total of 195 individuals consented to participate in the study. Eight (4.1\%) participants were omitted from the final analysis because they did not meet the criteria for study participation after self-identifying as intersex during the data collection procedure. Of those remaining in the sample $(N=187)$, participants were retained for analysis if they were missing $10 \%$ or less of the items for any given scale, survey, or tool.

\section{Results}

More participants identified as transmasculine $(45.0 \% ; \mathrm{n}=85)$ than transfeminine $(34.9 \%$; $\mathrm{n}=66)$ or nonbinary $(20.1 \% ; \mathrm{n}=38)$. Participant ages varied between 18 to $>65$, with most participants identifying as being between the ages of $26-35(n=63 ; 33.3 \%)$. Participants were predominantly white $(n=156 ; 82.5 \%)$ and non-Hispanic $(n=169 ; 89.4 \%)$. No participant identified as black or African American, although 24 (12.7\%) identified as mixed race and $2(1.1 \%)$ were unsure of race or unable to answer. Most participants had completed at least some college coursework $(n=67 ; 35.4 \%)$ or had earned a college degree $(n$ $=108 ; 57.2 \%$ ). Additional demographic data are presented in Table 1.

Table 1. Demographics (N=187)

\begin{tabular}{lrr}
\hline Variable & $\boldsymbol{n}$ & $\%$ \\
Gender & & \\
Transmasculine & 85 & 45.0 \\
Transfeminine & 66 & 34.9 \\
Nonbinary & 38 & 20.1 \\
& & \\
Age & & \\
$18-25$ & 42 & 22.2 \\
$26-35$ & 63 & 33.3 \\
$36-45$ & 41 & 21.7 \\
$46-55$ & 21 & 11.2 \\
$56-65$ & 11 & 5.8 \\
$>65$ & 11 & 5.8 \\
& & \\
Race & & \\
White & 154 & 82.4 \\
American Indian/Alaska Native & 4 & 2.1 \\
Asian & 3 & 1.6 \\
Mixed race & 24 & 12.8 \\
Do not know/unable to answer & 2 & 1.1 \\
& & \\
Ethnicity & & \\
Hispanic & 20 & 10.6 \\
Non-Hispanic & 169 & 89.4 \\
Educational Background & & \\
Less than high school & & \\
High school/Ged & 2 & 1.1 \\
Some college & 12 & 6.3 \\
College/University BS BA & 67 & 35.4 \\
Graduate degree & 54 & 28.6 \\
\hline
\end{tabular}


For participants providing geographic information $(\mathrm{n}=150 ; 80.2 \%)$, all resided in larger metro counties ( $n=141 ; 94.0 \%)$ or nonmetro counties with close geographic access to an urban population of 20,000 or more $(n=9 ; 6.0 \%)$ according to the rural-urban continuum 36. The majority $(n=166 ; 88.9 \%)$ reported having access to reliable transportation; less than $4 \%(n=6)$ reported never or rarely having access. Driving distance to transgender health specialists ranged from less than 10 miles $(n=75 ; 39.7 \%)$ to over 50 miles $(n=20$; $10.6 \%)$; however, the vast majority report traveling 25 miles or less $(\mathrm{n}=119 ; 71.75 \%)$. A little less than one-half $(n=86 ; 45.5 \%)$ reported waiting $1-2$ weeks to schedule an appointment with their provider; however, over half reported delays of 3 or more weeks (n $=103 ; 55.5 \%)$. Only a small number reported rarely or never having adequate health insurance $(\mathrm{n}=11 ; 5.8 \%$ and $\mathrm{n}=9 ; 4.7 \%$ respectively). The majority reported often $(\mathrm{n}=49$; $25.9 \%$ ) or always ( $\mathrm{n}=97 ; 51.3 \%)$ having adequate health insurance coverage.

The TFPKS was pilot tested in this study as a measure of TGD knowledge of fertility preservation. The mean number of knowledge points earned by participants $A M A B$ was $3.37(\mathrm{SD}=1.53)$, with a range of $0-7$; over $75 \%(n=52)$ of respondents from this group responded incorrectly to 3 or more of the 7 items. Knowledge points were similar for participants $A F A B$, earning a mean of 3.7 points $(S D=2.16)$ with almost $80 \%(n=95)$ selecting the correct response less than half of the time. Individual TFPKS items with participant responses are presented in Table 2.

Table 2. Transgender Fertility Preservation Knowledge Survey (TFPKS) Participant Responses

\begin{tabular}{|c|c|c|c|}
\hline Generic TFPKS Items & $\begin{array}{l}\text { True } \\
\mathrm{n}(\%)\end{array}$ & $\begin{array}{l}\text { False } \\
\mathrm{n}(\%)\end{array}$ & $\begin{array}{c}\text { Unsure } \\
\mathrm{n}(\%)\end{array}$ \\
\hline \multicolumn{4}{|c|}{ All gender transition treatment results in infertility. } \\
\hline All $\quad(N=180)$ & $9(4.8)$ & 142(75.1) & 29(15.3) \\
\hline $\operatorname{MAB}(n=68)$ & $6(8.85)$ & $52(76.5)$ & 10(14.7) \\
\hline $\mathrm{FAB}(n=111)$ & $3(2.7)$ & $89(81.1)$ & $18(16.7)$ \\
\hline \multicolumn{4}{|c|}{ You must be married or have a partner to receive fertility preservation } \\
\hline treatment. & $5(2.67)$ & 1321(70.4) & 42(22.2) \\
\hline All & $1(1.5)$ & $54(79.4)$ & $13(19.1)$ \\
\hline MAB & $4(3.6)$ & $79(71.2)$ & 28(25.24) \\
\hline \multicolumn{4}{|l|}{$\mathrm{FAB}$} \\
\hline \multicolumn{4}{|c|}{ All fertility preservation options have a similar chance of achieving } \\
\hline pregnancy. & $4(2.1)$ & $107(56.6)$ & $69(36.5)$ \\
\hline All & $3(4.4)$ & $37(55.9)$ & $27(39.7)$ \\
\hline MAB & $1(0.9)$ & 69(62.2) & $41(36.9)$ \\
\hline \multicolumn{4}{|l|}{$\mathrm{FAB}$} \\
\hline \multicolumn{4}{|c|}{ Insurance will not cover the cost of fertility preservation related to gen- } \\
\hline der transition. & $61(32.2)$ & 21(10.6) & $98(51.9)$ \\
\hline All & 19(27.7) & $7(11.8)$ & $41(60.3)$ \\
\hline MAB & $42(37.8)$ & $13(11.7)$ & $56(50.5)$ \\
\hline \multicolumn{4}{|l|}{$\mathrm{FAB}$} \\
\hline \multicolumn{4}{|c|}{ My fertility preservation options will be the same regardless of how } \\
\hline \multicolumn{4}{|c|}{ long I have been receiving hormonal treatment for gender transition or } \\
\hline at what age it was initiated. & $5(1.6)$ & $97(51.3)$ & $78(41.3)$ \\
\hline All & $2(2.9)$ & $37(54.4)$ & $29(42.6)$ \\
\hline
\end{tabular}




\begin{tabular}{|c|c|c|c|}
\hline Assigned Male at Birth TFPKS items & $\begin{array}{l}\text { True } \\
\mathrm{n}(\%)\end{array}$ & $\begin{array}{l}\text { False } \\
\mathrm{n}(\%)\end{array}$ & $\begin{array}{l}\text { Unsure } \\
\mathrm{n}(\%)\end{array}$ \\
\hline $\begin{array}{l}\text { An individual who has their sperm frozen will have it available for fu- } \\
\text { ture } \\
\quad \text { use whenever they are ready to pursue parenting options. }\end{array}$ & $43(50.0)$ & $7(10.34)$ & $27(39.7)$ \\
\hline $\begin{array}{l}\text { It is possible to freeze and store testicular tissue to generate sperm } \\
\text { in the future to pursue parenting options. }\end{array}$ & $8(11.8)$ & $9(13.2)$ & $51(75.0)$ \\
\hline Assigned Female at Birth TFPKS items & $\begin{array}{l}\text { True } \\
\mathrm{n}(\%)\end{array}$ & $\begin{array}{l}\text { False } \\
\mathrm{n}(\%)\end{array}$ & $\begin{array}{l}\text { Unsure } \\
\mathrm{n}(\%)\end{array}$ \\
\hline $\begin{array}{l}\text { Egg freezing has the same chance of pregnancy as embryo freez- } \\
\text { ing. }\end{array}$ & $2(1.8)$ & $39(35.1)$ & $70(63.1)$ \\
\hline $\begin{array}{l}\text { An individual who freezes their eggs will have them available in the } \\
\text { future whenever they are ready to pursue parenting options. }\end{array}$ & $57(51.4)$ & $8(7.2)$ & $465(41.4)$ \\
\hline Frozen eggs almost always result in a viable pregnancy. & $4(3.6)$ & $40(36.4)$ & $67(60.4)$ \\
\hline Frozen embryos almost always result in a viable pregnancy. & $12(10.8)$ & $28(25.20)$ & $710(64.0)$ \\
\hline
\end{tabular}

\section{Bold font indicates correct response}

Of the five 'generic' survey items (relevant regardless of gender assigned at birth), participants were most likely to correctly respond to the statement "All gender transition treatment results in infertility" as false $(n=142 ; 75.1 \%)$. They were least likely to be knowledgeable of insurance coverage specific to fertility preservation with fewer than $21 \%(n=7)$ of participants earning points for this item. Points earned for TFPKS items 15 did not vary between groups with participants identifying as AMAB earning 2.78 (SD $=1.35)$ points compared to AFAB participants earning $2.8(\mathrm{SD}=1.31)$ points $(\mathrm{t}(136)=-$ $.116 ; \mathrm{p}=.89)$. Further, younger participants, age 35 and under $(\mathrm{n}=96)$ did not earn more knowledge points than older participants $(n=81)$ regardless of gender assigned at birth $(\mathrm{M}=2.78 ; \mathrm{SD}=1.29$ versus $\mathrm{M}=2.80 ; \mathrm{SD}=1.35$ respectively $(\mathrm{t}(175)=-.776 ; \mathrm{p}=.91)$. Lastly, participants who had been offered a fertility preservation consult $(n=49)$ did not earn more knowledge points $(\mathrm{M}=2.98$; $\mathrm{SD}=1.22)$ than those not offered a fertility preservation consult $(\mathrm{n}=126)(\mathrm{M}=2.72 ; \mathrm{SD}=1.36)(\mathrm{t}(173)=1.15 ; \mathrm{p}=.89)$.

A comparison of total TFPS scores for AMAB and AFAB groups revealed a similar pattern. Younger $\mathrm{AMAB}$ participants $(\mathrm{M}=3.60 ; \mathrm{SD}=1.7)$ did not earn significantly more gender specific knowledge points than their older peers $(\mathrm{M}=3.24$; $\mathrm{SD}=1.41) \mathrm{t}(65)=.938$; $\mathrm{p}=.35)$. Findings were similar for AFAB participants, with younger participants earning on average 4.21 points $(\mathrm{SD}=2.0$ ) compared to older AFAB participants scores of 3.7 (SD $=1.83)$ vs. $\mathrm{M}=3.45) \mathrm{t}(108)=1.28 ; \mathrm{p}=.20$.

For AMAB participants, knowledge of cryopreservation was relatively low, with about half earning points for knowledge specific to cryopreservation of semen $(\mathrm{n}=34 ; 50.0 \%)$; far fewer earned points related to testicular tissue cryopreservation $(n=60 ; 8.2 \%)$. For AFAB participants, very few earned points specific to pregnancy success when comparing egg to embryo cryopreservation $(n=2 ; 1.8 \%)$; only $12(10.8 \%)$ earned points specific to embryo cryopreservation and achieving a successful pregnancy. Individual TFPKS 
items with participant responses are presented in Table 2. In early research, one can save time and money with instruments that have modest reliability; however, in applied settings where important decisions are made, a reliability of .90 is the minimum tolerated with a reliability of .95 being considered the standard (Nunnally, 1978). The TFPKS, created for this study, did perform at an acceptable level. Cronbach's alpha for TFPKS assigned male at birth (MAB) was .57 and .63 for those assigned female at birth (FAB). It is likely that minimal response variation to virtually all scale items limited the option of identify variation in knowledge levels as most items being responded to correctly or incorrectly by many of the participants. However, findings from this study indicate that it is likely the majority of TGD individuals undergo gender affirmation lacking the knowledge to make informed treatment 'decisions. To continue our work toward closing this care inequity, the TFPKS will be reviewed, revised, and re-tested with a larger more diverse group of young adults TGD individuals to create an improved TFPKS.

Most participants identified a patient-centered $(\mathrm{n}=129 ; 69.4 \%)$ or shared decision- making preference $(n=52 ; 27.5 \%)$ when making healthcare decisions (Table 3). For those reporting a physician centered preference $(n=5 ; 2.7 \%)$, most still identified the desire for their physician to "seriously consider my opinion" $(n=4 ; 2.2 \%)$. Despite this preference, only 20 participants $(10.6 \%)$ reported a belief that they were "Partners in care with their healthcare provider". Further, only 15 (7.9\%) reported having had their personal healthcare provider consistently ask them "about their healthcare preferences".

Table 3. Decision Making Preferences $(\mathrm{N}=186)$

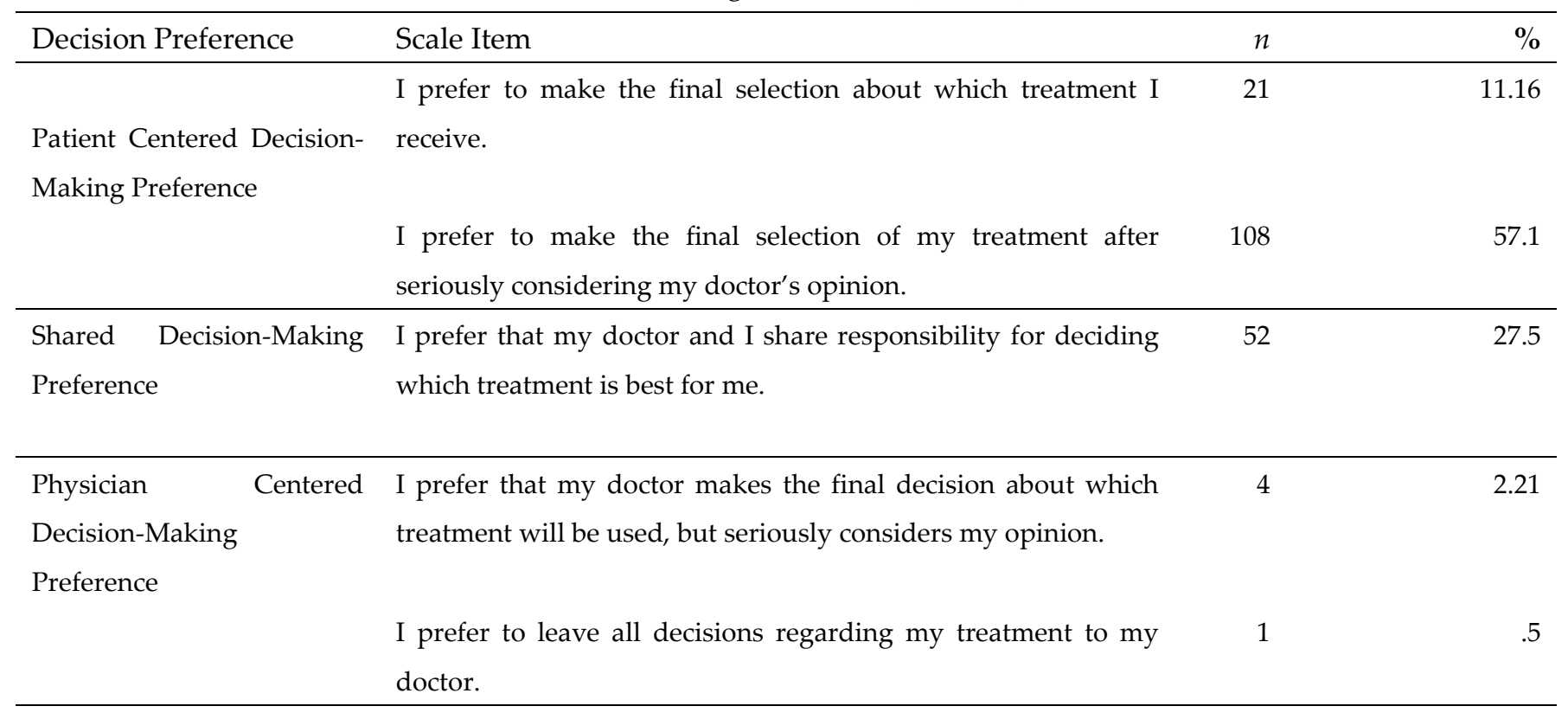

Only $27 \%(n=51)$ of participants report being offered fertility preservation counseling prior to medical transition; of these, only $6(3.2 \%)$ completed a consult. Participants providing reasons for not pursuing fertility preservation $(n=4)$ listed lack of financial resources and lack of interest in having children. Of those completing the consult, 3 elected to participate in fertility preservation treatment, 2 with semen cryopreservation and 1 with egg cryopreservation. All of those participating in fertility preservation treatment either "strongly agree" $(n=2)$ or "agree" $(n=1)$ that "It was the right decision" and that "The decision was a wise one". None regretted the healthcare decision made or thought the choice had done them harm. Of those who were not offered fertility preservation $(n=131)$, over $2 / 3(n=88 ; 67.2 \%)$ believed they should have been offered fertility preservation consultation and of these, 54 (41.2\%) state they would likely have participated in the consultation had it been offered. 
Participants were queried about their current interactions with personal healthcare providers. Over one-half of the time, participants reported either "often" ( $n=50 ; 26.5 \%)$ or "always" ( $\mathrm{n}=35 ; 18.5 \%)$ feeling their healthcare provider was "in need of training in transgender care". Further, consistently feeling as if their personal healthcare provider(s) exhibited culturally humility was identified by few participants $(\mathrm{n}=14 ; 7.4 \%)$ and the majority $(n=102 ; 57.3 \%)$ report delaying needed healthcare due to system and provider stigma.

Participants were provided with an opportunity to share any additional information related to their experiences with fertility preservation in an open text field. Rich comments were collected describing participant desire to participate in fertility preservation, their lack of interest in fertility preservation, and actual experiences with fertility preservation. Common comments included never having heard about fertility preservation, never having been offered a choice, and having made a personal decision to not have children prior to initiating affirmation treatment

\section{Discussion}

Transgender individuals represent a small but growing population in need of high-quality fertility preservation health information. Empowering patients to effectively engage in shared healthcare decision making is dependent upon patient access to accurate health information that supports an individual's informed understanding of their "health condition" and treatment options ${ }^{37}$. This includes an understanding of probable treatment outcomes and the opportunity to reflect upon how each treatment outcome might be individually experienced across the lifespan. Currently, there are no decision support tools are available to support TGD to make informed fertility preservation healthcare decisions ${ }^{38}$. While an internet search using the key words "transgender fertility preservation" will reveal multiple public and private websites containing TGD specific fertility preservation related information, content is limited in its ability to support high-quality shared healthcare decision making. This is problematic as TGD individuals are more likely to seek health information online due to the multiple barriers encountered in the healthcare system ${ }^{39}$. Decisions, such as fertility preservation, are supported by health information that supports good provider-patient communication, active patient participation, and the opportunity for patients to reflect upon personal preferences ${ }^{40}$. These components are critical when patients are facing difficult, life altering decisions, such as those faced at the time of gender affirmation ${ }^{41}$. As fertility preservation is considered a preference sensitive healthcare decision ${ }^{6-8}$, empowering the TGD person to identify and act upon their treatment preferences is pivotal in TGD patient fertility preservation counseling.

Reflecting national patterns ${ }^{42,43}$, participants frequently identified experiencing stigma and discrimination during healthcare encounters. As a result, TGD individuals may delay needed healthcare, seek health information from unreliable sources, or may never "come out" to their provider $39,44,45$. This is problematic and contributes to poor healthcare decision quality and subsequent decision regret.

An unexpected finding was the large number of participants engaging in gender affirmation medical care lacking knowledge of the potential impact on future fertility. Unfortunately, very few participants appear to have provided with the option to make informed decisions regarding fertility preservation. However, participation in fertility preservation within the TGD population must be considered within the context of gender affirmation medical treatment. Patient urgency to initiate gender affirmation 
treatment and the potential for increased gender dysphoria related to fertility preservation treatment are individual level barriers that may greatly influence participation ${ }^{46}$.

Study participants empowered to make an informed healthcare decision reported low levels of decision regret. However, this leaves a knowledge gap as little is known about decision regret in most participants related to the potential impact of gender affirmation medical care on future fertility and individual childbearing/parenting goals. Participant comments such as "I would have pursued the option if I knew more at the time" and "I didn't know it was an option at any point" may indicate high levels of decision regret regarding what may now be very limited fertility preservation options.

Knowledge scores were relatively low across the sample, regardless of age or assigned gender at birth. Participants were instructed not to "guess" on TFPKS items. If a participant "knew" the answer they were instructed to select that answer, if not, they were instructed to select "unsure". Across all TFPKS items, participants reported being "unsure" between $15.4 \%$ and $77.3 \%$ for each item. It is probable that knowledge points earned would be higher had participants been encouraged to select their "best guess". However, making high-quality health care decisions requires comprehensive health information, not best guesses.

While the knowledge base of fertility preservation health outcomes specific to gender affirmation treatment is nascent, fertility preservation within oncologic patients have been well described. Much of the early work in "onco-fertility" was related to young females diagnosed with breast or gynecologic cancer. Within this population, cancerrelated infertility was noted to cause significant levels of psychologic distress for over 2 decades 47,48 ; consideration of the impact of fertility on transgender persons has only recently garnered attention. However, the impact of gender affirmation treatment related infertility on psychologic distress and quality of life within the TGD population is largely unknown.

While our findings align with extant literature, much remains to be explored. Though healthcare professional are generally knowledgeable of the impact of gender affirmation medical treatment upon fertility, that knowledge of fertility does not always translate into fertility counseling ${ }^{11}$. Prevalent individual, provider, and healthcare system barriers may influence an individual's opportunity or desire to participate in fertility preservation counseling and/or treatment. These findings highlight the importance of developing fertility preservation health information specific to the needs of the TGD population, creating resources for practitioners to support fertility preservation counseling, and to ensure that TGD individuals have ready access to these resources.

\section{Implications}

Consistent with other studies, participants in our study identified healthcare provider specific barriers to care such as lack of cultural humility and limited expertise in TGD healthcare. Healthcare provider education has been posited as an effective method to address persistent health disparities within the TGD population ${ }^{49}$. Unfortunately, many healthcare professionals receive little if any TGD specific content within their preparatory educational programs ${ }^{50,51}$. Critical components of an effective education program would include content specific to gender identity and pedagogical interventions to inform attitudes toward TGD persons and the social and legal barriers prevalent in society ${ }^{52}$.

The need for healthcare professionals to support patient engagement in informed decision making has been a recognized standard of excellence for over 40 years ${ }^{53}$. One important facet of this mandate is to ensure access to high quality health information upon 
which to base treatment decisions and discussions. Unfortunately, health information resources such as decision aids do not appear to be available specific to the TGD population regarding fertility preservation. While multiple fertility preservation decisions aids specific to oncologic patients ${ }^{54-56}$ are readily available, application of a decision aid developed for use with a population facing a potentially fatal diagnosis would be inappropriate, as application to personal experience would not be possible. Further, decision aids for adolescent and young adult TGD individuals are currently being developed, information need to inform and adolescent and their parents is likely very different than that of the adult.

\section{Limitations}

Several limitations were identified during this study. First, the sample recruited was relatively homogenous with the vast majority identifying as "white". As a result, finding from this study may or may not represent those obtained from a more racially and ethnically diverse sample. An additional concern that arose during data analysis was the great diversity across participant age and the potential impact of history across time in participant responses. For example, a 60-year-old individual who transitioned 35 years ago may have limited memory of fertility preservation discussions.

While the use of social media to recruit during the Covid-19 pandemic has been identified as an effective method ${ }^{57}$, concerns regarding representativeness of the sample, determining the true response rate, and subsequent generalizability of the findings may arise ${ }^{58}$. Currently there is no conclusive evidence indicating a difference between selfcompleted paper surveys and online surveys 59 Effectiveness of online surveys using social media have proven effective in inaccessible populations ${ }^{60}$ and the use of this methodology is on the rise. likely to increase.

Research using self-report questionnaires may be plagued by response or social desirability bias ${ }^{61}$ confounding the research by creating false relationships or obscuring variables. Social desirability bias has been defined as the tendency to underreport socially undesirable attitudes and behaviors and to over report more desirable attributes ${ }^{62}$. However, totality of the evidence does not indicate a difference between online, offline, and paper surveys ${ }^{58}$. Given the anonymity of the survey and relatively low levels of study participants, and participant comments, response bias is unlikely. Lastly, the TFPKS may not have performed at the desired level of internal consistency due to low variation in participant responses and subsequent truncated response range. Exploring knowledge of fertility preservation and decision making in more diverse groups of transgender individuals is needed to inform the development of high-quality health information to better meet the needs of this highly diverse, at-risk population.

\section{Conclusions}

Clearly much remains to be done to meet the healthcare shared decision-making needs of the TGD population regarding fertility preservation. To address this gap, the development of a high-quality decision aid that provides relevant health information in addition to the opportunity to reflect upon how each treatment decision would be personally experienced is essential. A foundational step towards this action, is the identification of a valid and reliable instrument to measure TGD knowledge of fertility preservation. Toward this action, the TFPKS will be revised by researchers, TGD community and reproductive endocrinologist and re-tested with a racially and ethnically diverse sample of your adults (ages 19-35). These actions will help to support vulnerable TGD persons as they make potentially life altering healthcare decisions regarding gender affirmation and fertility preservation.

Author Contributions: All authors contributed equally to the research and writing of this article.

Funding: This study was not supported by any grant or other funding. 
Institutional Review Board Statement: “The study was conducted according to the guidelines of the Declaration of Helsinki, and approved by the Institutional Review Board of Montana State University on 10/21/2020 FWA\#00000165.

Informed Consent Statement: Informed consent was obtained from all subjects involved in the study.

Data Availability Statement: Montana State University is an institutional member of Dryad, and provides a DOI where readers can access the data. More information is available about data repositories and data availability on Montana State University library web site at the following link. Data Discovery - MSU Library | Montana State University

Acknowledgments: Authors would like to thank Trystan Reese of Collaborate Consulting for his expertise and personal experience with transgender fertility justice and the transgender community members involved in the development and execution of this study.

Conflicts of Interest: The authors declare no conflict of interest

\section{References}

1. Nolan IT, Dy GW, Levitt N. Considerations in Gender-Affirming Surgery: Demographic Trends. Urol Clin North Am. 2019;46(4):459-465.

2. Flores AR, Herman, J.L., Gates, G.J., \& Brown, T.N.T. How many adults identify as transgender in the United States. Los Angeles, CA: UCLA;2016.

3. Meyer IH, Brown TN, Herman JL, Reisner SL, Bockting WO. Demographic Characteristics and Health Status of Transgender Adults in Select US Regions: Behavioral Risk Factor Surveillance System, 2014. Am J Public Health. 2017;107(4):582-589.

4. Nolan IT, Kuhner CJ, Dy GW. Demographic and temporal trends in transgender identities and gender confirming surgery. Transl Androl Urol. 2019;8(3):184-190.

5. Waters E. Understanding healthcare for the transgender and gender non-conforming community. . In: Vermeesch A, ed. Integrative Health Nursing Interventions for Vulnerable Populations. Switzerland: Springer Nature 2021:89-107.

6. von Doussa H, Power J, Riggs D. Imagining parenthood: the possibilities and experiences of parenthood among transgender people. Cult Health Sex. 2015;17(9):1119-1131.

7. Mattawanon N, Spencer JB, Schirmer DA, 3rd, Tangpricha V. Fertility preservation options in transgender people: A review. Rev Endocr Metab Disord. 2018;19(3):231-242.

8. Morrison A, Olezeski, C., Cron, J. , Kallen, N. A pilot study to assess attitudes toward fuure fertility and parenthood in trangender and gender expansive adolescents. . Transgender Health. 2020;5(2):129-137.

9. Elwyn G, O'Connor AM, Bennett C, et al. Assessing the quality of decision support technologies using the International Patient Decision Aid Standards instrument (IPDASi). PLoS One. 2009;4(3):e4705.

10. World Professional Association for Transgender Health. Standards of care for the health of transsexual, transgender, and gender-nonconforming people. . In: 7th ed.2012: https://www.wpath.org/publications/soc.

11. Chen D, Kolbuck VD, Sutter ME, Tishelman AC, Quinn GP, Nahata L. Knowledge, Practice Behaviors, and Perceived Barriers to Fertility Care Among Providers of Transgender Healthcare. J Adolesc Health. 2019;64(2):226-234.

12. Vyas N, Douglas CR, Mann C, Weimer AK, Quinn MM. Access, barriers, and decisional regret in pursuit of fertility preservation among transgender and gender-diverse individuals. Fertil Steril. 2020.

13. Brockting W, Coleman, E., De Cuypere, G. Care of transsexual persons. New England Journal of Medicine. 2011;364(26):2559-2660.

14. Wallace SA, Blough KL, Kondapalli LA. Fertility preservation in the transgender patient: expanding oncofertility care beyond cancer. Gynecol Endocrinol. 2014;30(12):868-871.

15. De Roo C, Tilleman K, T'Sjoen G, De Sutter P. Fertility options in transgender people. Int Rev Psychiatry. 2016;28(1):112-119.

16. Murad MH, Elamin MB, Garcia MZ, et al. Hormonal therapy and sex reassignment: a systematic review and meta-analysis of quality of life and psychosocial outcomes. Clin Endocrinol (Oxf). 2010;72(2):214-231.

17. Nahata L, Chen D, Moravek MB, et al. Understudied and Under-Reported: Fertility Issues in Transgender Youth-A Narrative Review. J Pediatr. 2019;205:265-271.

18. Keirns CC, Goold SD. Patient-centered care and preference-sensitive decision making. JAMA. 2009;302(16):1805-1806.

19. Health WPAfT. Standards if care for the health of transexual, Transgender, and Gender-Nonconforming People In:2012: https://www.wpath.org/media/cms/Documents/SOC\%20v7/SOC\%20V7_English2012.pdf?_t=1613669341.

20. Hembree WC, Cohen-Kettenis PT, Gooren L, et al. Endocrine Treatment of Gender-Dysphoric/Gender-Incongruent Persons: An Endocrine Society Clinical Practice Guideline. Endocr Pract. 2017;23(12):1437.

21. Chen D, Simons L, Johnson EK, Lockart BA, Finlayson C. Fertility Preservation for Transgender Adolescents. J Adolesc Health. 2017;61(1):120-123.

22. Brehaut JC, O'Connor AM, Wood TJ, et al. Validation of a decision regret scale. Med Decis Making. 2003;23(4):281-292.

23. Bartholomaeus C, \& Riggs, D. Transgender and non-binary Australians experience with healthcare professionals in relation to fertilty preservation Culture, Health and Sexuality 2019;22(2):129-145. 
24. Auer MK, Fuss J, Nieder TO, et al. Desire to Have Children Among Transgender People in Germany: A Cross-Sectional MultiCenter Study. J Sex Med. 2018;15(5):757-767.

25. Ainsworth AJ, Allyse M, Khan Z. Fertility Preservation for Transgender Individuals: A Review. Mayo Clin Proc. 2020;95(4):784792.

26. Adeleye AJ, Reid G, Kao CN, Mok-Lin E, Smith JF. Semen Parameters Among Transgender Women With a History of Hormonal Treatment. Urology. 2019;124:136-141.

27. Dunne P. Transgender Sterilisation Requirements in Europe. Med Law Rev. 2017;25(4):554-581.

28. De Sutter P, KIra, K., Verschoor, A., Hotimsky, A., De Sutter, P., . The desire to have children and preservation of fertility in transexual. women: A survey. The International Journal of Transgenderism 2002;6(3).

29. Wierckx K, Stuyver I, Weyers S, et al. Sperm freezing in transsexual women. Arch Sex Behav. 2012;41(5):1069-1071.

30. Wierckx K, Van Caenegem E, Pennings G, et al. Reproductive wish in transsexual men. Hum Reprod. 2012;27(2):483-487.

31. Miller CC. American's are having few babies. They told us why. The New York Times 2018.

32. Degner LF, Sloan JA, Venkatesh P. The Control Preferences Scale. Can J Nurs Res. 1997;29(3):21-43.

33. Singh JA, Sloan JA, Atherton PJ, et al. Preferred roles in treatment decision making among patients with cancer: a pooled analysis of studies using the Control Preferences Scale. Am J Manag Care. 2010;16(9):688-696.

34. Pasick R, Geraldine, O., Goldstein, E., Nguyen, T. Community engaged research with community based organizations. A resource manual for researchers. . In: Fleisher P, ed. University of California, San Francisco2010: https://accelerate.ucsf.edu/files/CE/manual_for_researchers_agencies.pdf. Accessed March 21, 2021.

35. Kongats K, Wright MT. Participatory Health Research : Voices from Around the World. In: 1st ed. Cham: Springer International Publishing : Imprint: Springer,; 2018.

36. United States Department of Agriculture. Rural-Urban continuum codes 2013.

37. Lewis CL, Pignone MP. Promoting informed decision-making in a primary care practice by implementing decision aids. $N$ C Med J. 2009;70(2):136-139.

38. Kolbuck VD, Sajwani A, Kyweluk MA, Finlayson C, Gordon EJ, Chen D. Formative development of a fertility decision aid for transgender adolescents and young adults: a multidisciplinary Delphi consensus study. J Assist Reprod Genet. 2020;37(11):28052816.

39. Jabson JM, Patterson JG, Kamen C. Understanding Health Information Seeking on the Internet Among Sexual Minority People: Cross-Sectional Analysis From the Health Information National Trends Survey. JMIR Public Health Surveill. $2017 ; 3(2):$ e39.

40. Bae JM. Shared decision making: relevant concepts and facilitating strategies. Epidemiol Health. 2017;39:e2017048.

41. Elwyn G, Frosch D, Volandes AE, Edwards A, Montori VM. Investing in deliberation: a definition and classification of decision support interventions for people facing difficult health decisions. Med Decis Making. 2010;30(6):701-711.

42. Center for Disease Control and Prevention. Patient-centered care for transgender people: Recommended practices for healthcare settings. . 2020. https://www.cdc.gov/hiv/clinicians/transforming-health/health-care-providers/affirmative-care.html. Accessed March 21, 2021.

43. Grant JM, Motter, L.A., Tanis, J., Harrison, J., Herman, J.L., \& Keisling, M. . Injustice at every turn: A report of the National Transgender Discrimination Survey 2011. https://dataspace.princeton.edu/handle/88435/dsp014j03d232p. Accessed March 21.

44. Safer JD. Hurdles to health care access for transgender individuals. Nat Hum Behav. 2019;3(11):1132-1133.

45. Safer JD. Transgender Patients And Health Care Providers. Health Aff (Millwood). 2017;36(12):2213.

46. Tishelman AC, Sutter ME, Chen D, et al. Health care provider perceptions of fertility preservation barriers and challenges with transgender patients and families: qualitative responses to an international survey. J Assist Reprod Genet. 2019;36(3):579-588.

47. Carter J, Rowland K, Chi D, et al. Gynecologic cancer treatment and the impact of cancer-related infertility. Gynecol Oncol. 2005;97(1):90-95.

48. Byrne J. Infertility and premature menopause in childhood cancer survivors. Med Pediatr Oncol. 1999;33(1):24-28.

49. Mayer KH, Bradford JB, Makadon HJ, Stall R, Goldhammer H, Landers S. Sexual and gender minority health: what we know and what needs to be done. Am J Public Health. 2008;98(6):989-995.

50. Obedin-Maliver J, Goldsmith ES, Stewart L, et al. Lesbian, gay, bisexual, and transgender-related content in undergraduate medical education. JAMA. 2011;306(9):971-977.

51. F, Johnson M, Eliason M. A National Survey of Faculty Knowledge, Experience, and Readiness for Teaching Lesbian, Gay, Bisexual, and Transgender Health in Baccalaureate Nursing Programs. Nurs Educ Perspect. 2015;36(3):144-152.

52. Dubin SN, Nolan IT, Streed CG, Jr., Greene RE, Radix AE, Morrison SD. Transgender health care: improving medical students' and residents' training and awareness. Adv Med Educ Pract. 2018;9:377-391.

53. United States. President's Commission for the Study of Ethical Problems in Medicine and Biomedical and Behavioral Research. Making health care decisions : a report on the ethical and legal implications of informed consent in the patient-practitioner relationship. Washington, D.C.: President's Commission for the Study of Ethical Problems in Medicine and Biomedical and Behavioral Research : For sale by the Supt. of Docs., U.S. G.P.O.; 1982.

54. Benoit A, Grynberg M, Morello R, Sermondade N, Grandazzi G, Moutel G. Does a web-based decision aid improve informed choice for fertility preservation in women with breast cancer (DECISIF)? Study protocol for a randomised controlled trial. BMJ Open. 2020;10(2):e031739.

55. Garvelink MM, ter Kuile MM, Fischer MJ, et al. Development of a Decision Aid about fertility preservation for women with breast cancer in The Netherlands. J Psychosom Obstet Gynaecol. 2013;34(4):170-178. 
56. Hamano I, Hatakeyama S, Ohyama C. Fertility preservation of patients with testicular cancer. Reprod Med Biol. 2017;16(3):240251.

57. Ali SH, Foreman J, Capasso A, Jones AM, Tozan Y, DiClemente RJ. Social media as a recruitment platform for a nationwide online survey of COVID-19 knowledge, beliefs, and practices in the United States: methodology and feasibility analysis. BMC Med Res Methodol. 2020;20(1):116.

58. Dodou D, Winter, J.C. Social desirability is the same in offline, online and paper surveys: A meta-analysis. . Computers in Human Behavior. 2014;36:487-495.

59. Lefever S, Dal, M., Matthiasdottir, A.,. Online data collection in academic research: Advantages and limitations. British Journal of Educational Technology. 2007;38(4):574-582.

60. Gelinas L, Pierce R, Winkler S, Cohen IG, Lynch HF, Bierer BE. Using Social Media as a Research Recruitment Tool: Ethical Issues and Recommendations. Am J Bioeth. 2017;17(3):3-14.

61. van de Mortel TF. Faking it: Social desirability response bias in self-report research. . Austrailan Journal of Advnanced Nursing. 2008;25(4):40-48.

62. Latkin CA, Edwards C, Davey-Rothwell MA, Tobin KE. The relationship between social desirability bias and self-reports of health, substance use, and social network factors among urban substance users in Baltimore, Maryland. Addict Behav. 2017;73:133-136. 